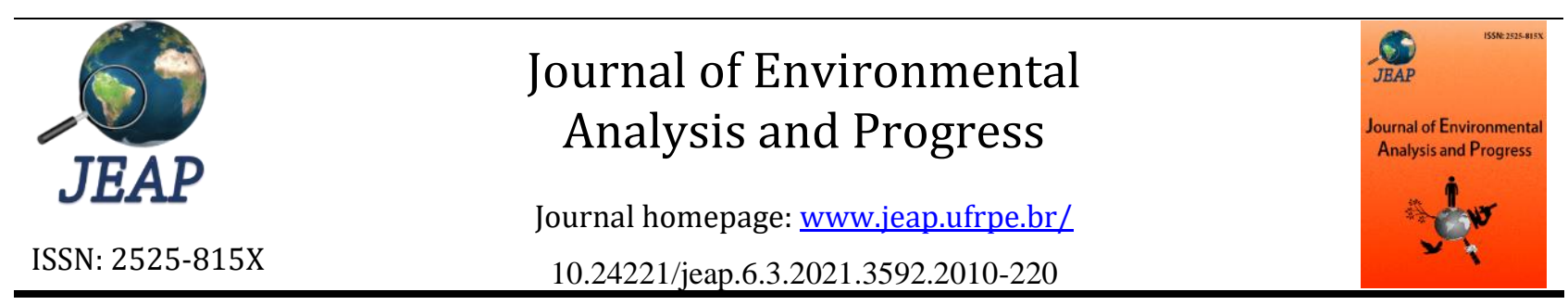

\title{
Beneficiamento de jeans no Agreste pernambucano e os gaps entre a situação real e ideal, fundamentada pelo método da Produção Mais Limpa
}

\section{Processing of jeans in Agreste of Pernambuco and the gaps between the real and ideal situation, based on the Cleaner Production method}

\author{
Maria de Fátima da Silva ${ }^{\mathrm{a}}$, Ana Regina Bezerra Ribeiro ${ }^{\mathrm{b}}$, Almir Silveira Menelau ${ }^{\mathrm{c}}$ \\ a Universidade Federal Rural de Pernambuco-UFRPE. Rua Dom Manoel de Medeiros, SN, Dois Irmãos, Recife, \\ Pernambuco, Brasil. CEP: 52171-900. E-mail: fatima.professora.adm@gmail.com, anaregina.ribeiro@ufrpe.br, \\ almirmenelau@yahoo.com.br.
}

A R T I CLE I N F O

Recebido 03 Jun 2020

Aceito 23 Abr 2021

Publicado 07 Set 2021

\begin{abstract}
A B S T R A C T
The Cleaner Production - $\mathrm{C}+\mathrm{P}$ is a preventive environmental strategy that must be applied continuously in processes, products, and services to avoid ecological problems at the source and generating economic, social, and environmental advantages for the organization. According to Cleaner Production in jeans textile laundries in Agreste region of Pernambuco, the study aimed to measure the gaps between the ideal situation and the actual situation. The research is characterized as a field with descriptive quantitative data analysis with the aid of SPSS software. As a result, it is noteworthy that the gaps in knowledge of the $\mathrm{C}+\mathrm{P}$ tool, production process, and customer are the highlights. It is concluded that there are gaps in all the dimensions analyzed in the textile laundries concerning $\mathrm{C}+\mathrm{P}$, highlighting the need to understand $\mathrm{C}+\mathrm{P}$ for all involved and the adoption of educational policies aimed at sustainability for the region in question study.
\end{abstract}

Keywords: Textile sector, sustainable method, productive arrangement.

\begin{abstract}
R E S U M O
A Produção Mais Limpa - P+L é uma estratégia ambiental preventiva que deve ser aplicada de forma contínua nos processos, produtos e serviços visando evitar problemas ambientais na fonte e gerando vantagens econômicas, sociais e ambientais para a organização. O estudo objetivou mensurar os gaps entre a situação ideal e a situação real de acordo com a $\mathrm{P}+\mathrm{L}$ nas lavanderias têxteis de jeans no Agreste Pernambucano. A pesquisa é caracterizada como de campo, com análise de dados quantitativos, descritiva, com auxílio do software SPSS. Destaca-se que os gaps como: conhecimento da ferramenta $\mathrm{P}+\mathrm{L}$, processo produtivo e cliente são os de maior destaque. Conclui-se que existem lacunas em todas as dimensões analisadas nas lavanderias têxteis em relação à $\mathrm{P}+\mathrm{L}$, destacam-se a necessidade da compreensão da $\mathrm{P}+\mathrm{L}$ para todos os envolvidos bem como adoção de políticas educacionais que visem o desenvolvimento sustentável da região estudada.

Palavras-Chave: Setor têxtil, método sustentável, arranjo produtivo.
\end{abstract}

\section{Introdução}

O processo do beneficiamento têxtil é caracterizado pelo alto consumo de água e utilização química de produtos como tensoativos, amaciantes, alvejantes, tinturas, entre outros. No entanto, são poucas as empresas que se preocupam em lançar seus efluentes corretamente. Assim, as lavanderias têxteis são consideradas como atividades insalubres devido ao alto emprego de substâncias químicas e operarem em áreas molhadas e com altas temperaturas (Buss et al., 2015).

As etapas convencionais do beneficiamento do jeans são consideradas de alta potencialidade negativa à saúde da humanidade, pois envolve substâncias altamente tóxicas. Neste sentido, o procedimento de lavagem e tingimento do jeans é responsável pela geração de efluentes e resíduos, além da poluição do corpo hídrico, que vem modificando o solo (Lopes, 2011). 
Este processo também promove a poluição atmosférica, pela utilização de chaminés sem filtros, degradação do espaço em virtude da destinação incorreta dos resíduos, refletindo, negativamente, na condição da vida social, além de dificultar os surgimentos de novas indústrias (Silva Filho, 2003).

De acordo com Nascimento (2021), em todo o mundo são descartadas toneladas de materiais têxteis em aterros sanitários todos os dias. Estima-se que a cada segundo é gerado um caminhão de lixo da indústria do vestuário.

O Brasil descarta resíduos têxteis em uma taxa alarmante a cada ano, o que é muito prejudicial ao meio ambiente. Em 2014, a indústria brasileira gerou, aproximadamente, 170.000 toneladas de resíduos, que acabaram ficando sem uso e foram depositados em aterros sanitários (Amaral et al, 2018). De 2017 a 2020 foram geradas 29.169 toneladas de resíduos têxteis, dos quais, mais de $80 \%$ foram oriundos da produção de confecções (Nascimento, 2021).

Araújo (2015) acrescenta que o gerenciamento dos resíduos representa um dos problemas mais críticos da atualidade. O descarte, de forma inadequada, dos resíduos, pode resultar na poluição urbana e rural.

Nesse intuito, as lavanderias têxteis têm contribuído de forma significativa para a deterioração do espaço ambiental e muitos dos empresários acreditam que adotar métodos de cuidados ambientais provoca custos extras, e que medidas como redução de água, energia e matériaprima são apenas ações que proporcionam economia de recursos, não atentando para o fato da geração de benefícios financeiros, devido a prevenção de acidentes, prejuízos e multas decorrentes de agressões socioambientais (Bezerra \& Monteiro, 2009; Bezerra \& Freitas, 2013).

Pode-se afirmar que, as políticas de responsabilidade ambiental vêm evoluindo dos métodos tradicionais de tratamento para os princípios de prevenção da poluição. Nesse aspecto, fundamenta-se a Produção Mais Limpa $(\mathrm{P}+\mathrm{L})$, a qual tem a intenção de reduzir os resíduos na fonte, aumentando a eficiência no emprego dos recursos e evitam, ao máximo, o desperdício de insumos na produção.

Nesse entendimento, a $\mathrm{P}+\mathrm{L}$ contribui com a credibilidade da organização por meio da valorização de sua imagem, redução de multas e penalidades, eficiência produtiva e comprometimento socioambiental (Silva, Fritsch \& Silva, 2018). Assim, ao investir em $\mathrm{P}+\mathrm{L}$, a empresa consegue garantir a competitividade com as demais empresas do mesmo ramo (Silva, Fritsch \& Silva, 2018).
Para Silva \& Menelau (2021), a P+L é um modelo produtivo que versa sobre uma mudança na cultura organizacional da empresa, por meio de hábitos sustentáveis que garanta atender a legislação ambiental e proporcione ganhos econômico, ambiental e social.

Assim, as práticas de $\mathrm{P}+\mathrm{L}$ podem melhorar os processos produtivos, minimizar impactos ambientais negativos e, consequentemente, aumentar a vantagem competitiva das organizações. Diante do exposto, o estudo objetivou mensurar os gaps entre a situação ideal e a situação real, de acordo com a $\mathrm{P}+\mathrm{L}$ nas lavanderias têxteis de jeans no Agreste de Pernambuco.

\section{Material e Métodos}

Durante os meses de julho a outubro de 2019 foram coletadas informações sobre os processos produtivos e gerenciais das lavanderias têxteis no Arranjo Produtivo do Agreste Pernambucano-APL.

O universo da 'pesquisa é composto por 161 lavanderias têxteis, localizadas nos cinco municípios que constituem o Arranjo Produtivo de Confecções do Agreste Pernambucano, em Caruaru, Toritama, Vertentes, Surubim e Riacho das Almas.

O estudo é classificado como descritivo; esse tipo de investigação permite descrever as características de populações ou fenômenos, por meio de técnicas de coletas de dados padronizadas, como questionário e observação sistemática (Gil, 2008).

Nesse sentido, Malhotra (2012) afirma que o questionário com perguntas diretas é um importante instrumento da pesquisa descritiva. Vale salientar que a análise, com base em questionário, considera verídicas as respostas dos respondentes.

$\mathrm{O}$ estudo configurou-se, de acordo com Vergara (2010), como de pesquisa de campo; este tipo de estudo corresponde à coleta de dados, observações, análise e interpretação de fenômenos e de fatos que ocorrem ou ocorreram na amostra. Fonseca (2002) corrobora ao afirmar que, a pesquisa de campo se diferencia das demais investigações, principalmente das pesquisas bibliográfica e documental, visto que é feita com o apoio da coleta de dados junto a pessoas/empresas.

Quanto aos fins, a pesquisa é descritiva e exploratória que, segundo Gil (2008), tem a intenção de proporcionar maior familiaridade com o problema analisado e torná-lo mais explícito.

Como principal estratégia para a coleta de dados, utilizou-se o questionário, por possibilitar 
uma avaliação mais ampla do processo existente nas indústrias de beneficiamento do jeans.

Para calcular o grau de significância da amostra, utilizou-se a Equação 1.

$$
\sum \frac{\frac{z^{2} \cdot p(1-p)}{e^{2}}}{1+\left(\frac{z^{2} \cdot p(1-p)}{e^{2} \cdot N}\right)}
$$

onde $\mathrm{N}=161$ lavanderias (população); e $=0,10$ (margem de erro); $\mathrm{Z}=1,65$ (escore $\mathrm{z}$ ); $\mathrm{p}=0,9$ (nível de significância).

Após o cálculo da amostra, ficou evidente que seriam necessárias 31,68 empresas (32 lavanderias) para representar uma amostra estatisticamente relevante. Por segurança, acrescentou-se mais seis lavanderias, ou seja, acrescentou, aproximadamente, $20 \%$ da amostra, totalizando uma amostra final de 38 lavanderias.

Vale ressaltar que, a rigor, a maioria das pesquisas operam com uma significância da amostra entre $95 \%$ e $99 \%$. Isto acontece, porque ocorre em função do grau de controle que se estabelece sobre a população/amostra. No caso em análise, se impôs $90 \%$, pois tornou-se impossível estabelecerem-se controles sobre a população/amostra.

$\mathrm{Na}$ verdade, configurou-se o que se pode chamar de amostra de conveniência, em fase de somente ter sido possível convencer 38 dirigentes de organizações (lavanderias) a participarem do estudo; para assegurar tal número, se impôs uma significância de $90 \%$.

Todavia, foi acrescentado à amostra mais $20 \%$ de organizações, o que chegou ao nível relevante de significância. Conforme Patino \& Ferreira (2015), o intervalo de confiança de $95 \%$ é o mais comum dos intervalos relatados na literatura. No entanto, é possível usar intervalos de confiança de $90 \%$ ou $99 \%$, caso se deseje mais ou menos confiança.

$\mathrm{O}$ intervalo de confiança representa a incerteza do tamanho do efeito na população de origem, e não na população de estudo. Assim, o cálculo do intervalo de confiança tem seu tamanho apontado pelo tamanho da amostra, erro e grau de confiança, ou seja, os dirigentes que aceitaram participar da pesquisa (Patino \& Ferreira, 2015).

A seleção das lavanderias foi realizada de forma aleatória e foi composta a partir das disponibilidades dos proprietários. O questionário utilizou-se de uma escala de cinco gradações, que variaram de discordo totalmente (nível 1) até ao concordo totalmente (nível 5), com afirmativas relacionadas à $\mathrm{P}+\mathrm{L}$, referente à organização, ao uso de matéria-prima e de tecnologia, aos clientes, aos fornecedores, ao ambiente externo, à legislação ambiental, ao processo, à saúde do trabalhador e ao comprometimento socioambiental.

A análise de dados foi realizada de forma quantitativa, com auxílio do software Statistical Package for the Social Science - SPSS (Battisti et al., 2015).

A mensuração dos gaps da $\mathrm{P}+\mathrm{L}$ foi definida com base em 11 dimensões, sendo seis internas e cinco externas à organização, conforme Quadros 1 e 2.

As dimensões internas analisadas foram: Gestão Organizacional; Processo; Gestão de Resíduos; Conhecimento do a P+L; Saúde do trabalhador e Colaborador, tais dimensões foram elaboradas com base no CNTL e estudos recentes sobre a P+L no Brasil e no mundo (Quadro 1).

Quadro 1. Definição das dimensões internas da representação polar da P+L. Fonte: Silva, Ribeiro \& Menelau (2019).

\begin{tabular}{|l|l|l|}
\hline Dimensões internas & Definição dos estudos sobre a P+L & Estudos \\
\hline Gestão organizacional & $\begin{array}{l}\text { Como planeja a produção, lidera o pessoal } \\
\text { envolvido e controla os resultados } \\
\text { econômicos, sociais e ambientais. }\end{array}$ & $\begin{array}{l}\text { CNTL (2003); Fonseca \& Martins } \\
\text { (2018); Fresner \& Krem (2018); } \\
\text { Quevedo \& Paganini (2017); Silva } \\
\text { \& Menelau (2021). }\end{array}$ \\
\hline \multirow{3}{*}{ Processo } & $\begin{array}{l}\text { Reciclagem e reuso no ambiente interno, } \\
\text { modificações tecnológicas no sistema } \\
\text { produtivo e foco na adoção de tecnologias } \\
\text { limpas preventiva. }\end{array}$ & $\begin{array}{l}\text { CNTL (2003); Mohammad et al. } \\
\text { (2017); Rahman et al. (2017); } \\
\text { Silva, Fritsch \& Silva (2018). }\end{array}$ \\
\hline Gestão de Resíduos & $\begin{array}{l}\text { Armazenagem e destinação correta dos } \\
\text { elementos não reciclados, redução e } \\
\text { controle das emissões e resíduos líquidos } \\
\text { (efluentes), sólidos e gasosos. }\end{array}$ & $\begin{array}{l}\text { Quevedo \& Paganini (2017); } \\
\text { Franco \& Arias (2018); Silva, } \\
\text { Fritsch \& Silva (2018); CNTL } \\
\text { (2003). }\end{array}$ \\
\hline Conhecer a P+L & $\begin{array}{l}\text { Conhecer a P+L é ponto fundamental para } \\
\text { o sucesso da gestão. }\end{array}$ & $\begin{array}{l}\text { CNTL (2003); Costa et al. (2014); } \\
\text { Silva, Moraes \& Machado (2015). }\end{array}$ \\
\hline
\end{tabular}




\begin{tabular}{|l|l|l|}
\hline \multirow{5}{*}{ Saúde do trabalhador } & $\begin{array}{l}\text { Contribuir para a integridade física e } \\
\text { mental dos colaboradores, além de } \\
\text { proporcionar equipamentos corretos para } \\
\text { sua proteção no ambiente de trabalho. }\end{array}$ & $\begin{array}{l}\text { Machado (2015); Quevedo \& } \\
\text { Paganini (2017); Passini, Chagas \& } \\
\text { Demarco (2019). }\end{array}$ \\
\hline Colaborador & $\begin{array}{l}\text { Treinamentos em educação ambiental, } \\
\text { desenvolvimento de habilidades e e } \\
\text { capacidade necessária para evitar } \\
\text { desperdício de recursos. }\end{array}$ & $\begin{array}{l}\text { CNTL (2003); Silva, Moraes \& } \\
\text { Paganini (2015); Quevedo \& }\end{array}$ \\
\hline
\end{tabular}

No Quadro 2 pode-se identificar as dimensões externas à organização que, segundo estudos, são importantes para o sucesso da P+L em qualquer organização, além de afirmarem que as práticas de $\mathrm{P}+\mathrm{L}$ contribuem para o equilíbrio da organização no uso da matéria-prima, no relacionamento com o cliente e fornecedor, no ambiente externo e nas políticas e legislação.

Quadro 2. Definição das dimensões externas da representação polar da Produção Mais Limpa - P+L. Fonte: Silva, Ribeiro \& Menelau (2019).

\begin{tabular}{|c|c|c|}
\hline $\begin{array}{l}\text { Dimensões } \\
\text { externas }\end{array}$ & Definição sobre a $\mathbf{P}+\mathbf{L}$ & Estudos \\
\hline Cliente & $\begin{array}{l}\text { Participação e cooperação na mudança de processo e } \\
\text { produto, visando atribuir fatores ambientais que } \\
\text { minimizem o impacto negativo ao meio ambiente e à } \\
\text { saúde humana. }\end{array}$ & $\begin{array}{l}\text { CNTL (2003); Mauser et al. } \\
\text { (2014); Passini, Chagas \& } \\
\text { Demarco (2019). }\end{array}$ \\
\hline $\begin{array}{l}\text { Matéria- } \\
\text { prima }\end{array}$ & $\begin{array}{l}\text { Uso consciente da água, da energia, de produtos } \\
\text { químicos e da madeira, com o objetivo de diminuir, } \\
\text { controlar e substituir seu uso na produção. }\end{array}$ & $\begin{array}{l}\text { CNTL (2003); Passini, Hagas \& } \\
\text { Demarco (2019); Rahman et al. } \\
\text { (2017). }\end{array}$ \\
\hline Fornecedor & $\begin{array}{l}\text { Participação e cooperação na mudança de processo, a } \\
\text { partir do fornecimento de matérias primas e } \\
\text { componentes ecológicos. }\end{array}$ & $\begin{array}{l}\text { CNTL (2003); Yukseler et al. } \\
\text { (2017). }\end{array}$ \\
\hline $\begin{array}{l}\text { Ambiente } \\
\text { externo }\end{array}$ & Melhoria na imagem da empresa & $\begin{array}{l}\text { Silva, Moraes \& Machado } \\
\text { (2015). }\end{array}$ \\
\hline $\begin{array}{l}\text { Políticas e } \\
\text { Legislação }\end{array}$ & $\begin{array}{l}\text { Atendimento às normas legais, evitando multas e } \\
\text { advertências. }\end{array}$ & $\begin{array}{l}\text { CNTL (2003); Silva, Moraes \& } \\
\text { Machado (2015); Quevedo \& } \\
\text { Paganini (2017). }\end{array}$ \\
\hline
\end{tabular}

A representação polar apresenta escores entre 1,0 e 5,0, classificados como: 1,0 (empresa discorda totalmente das práticas de $\mathrm{P}+\mathrm{L}$ ); 2,0 (a empresa discorda das práticas de $\mathrm{P}+\mathrm{L}$ ) 3,0 (a empresa é neutra as práticas de $\mathrm{P}+\mathrm{L}$ ); 4,0 (a empresa concorda parcialmente com as práticas de $\mathrm{P}+\mathrm{L}$ ) ou 5,0 (a empresa concorda totalmente com as práticas de $\mathrm{P}+\mathrm{L}$ ). Para calcular os gaps por dimensões utilizou-se a soma total das variáveis, dividida pelo total de respondentes (média).

Os resultados que mais aproximam-se do escore 5 são considerados, neste estudo, como menor gap, ou seja, mais próximo de um, e é considerado de maior gap ao comparar a produção atual das lavanderias têxteis do Agreste pernambucano em relação à produção ideal, a partir do método da $\mathrm{P}+\mathrm{L}$.

O Excel 2013 foi uma das ferramentas utilizadas para a tabulação dos dados e confecção dos gráficos, tabelas e quadros. As correlações de Spearman foram confeccionadas com o software SPSS.
Para o cálculo de Spearman foi usada a Equação 2.

$$
p=1-\frac{6 \sum_{i=1}^{n} d_{i}^{2}}{n^{3}-n}
$$

onde $\mathrm{n}=$ quantidade de pares $(\mathrm{xi}, \mathrm{yi})$ e di $=$ (postos de xi dentre os valores de $\mathrm{x}$ ) - (postos de yi dentre os valores de y).

Se os pontos de $\mathrm{x}$ são exatamente iguais aos pontos de y, então todos os di serão zero e $\rho$ será 1 . O $\rho$ valor de Spearman varia entre -1 e 1 .

Assim, os resultados próximos dos extremos são considerados de maior associação entre as variáveis, seja positivo ou negativo. Deste modo, o sinal negativo da correlação significa que as variáveis variam em sentido contrário, isto é, as categorias mais elevadas de uma variável estão associadas às categorias mais baixas da outra variável.

Conforme Pestana \& Gageiro (2003), os p com valores entre 0,01 e 0,39 são considerados como associações baixas; entre 0,4 e 0,69 são 
associações moderadas; entre 0,7 e 11 são consideradas associações altas.

\section{Resultados e Discussão}

O arranjo produtivo de confecção do Agreste de Pernambuco é composto por 10 cidades, porém, as empresas de beneficiamento do jeans concentram-se no município de Toritama e circunvizinhos como: Caruaru, Vertentes, Surubim e Riacho das Almas que juntos totalizam 161 empresas de beneficiamento de jeans.

Este tipo de empreendimento iniciou-se no município de Toritama na década de 1980 com a Lavanderia Jussara, tendo o empreendedor Caló Pantaleon (italiano) como pioneiro na área de beneficiamento do jeans. A partir deste, novos empreendimentos foram surgindo nos municípios e nos circunvizinhos para atender a demanda da confecção de jeans do Agreste pernambucano.

Outra característica da região é a ocupação da maioria da população no segmento de confecção, a exemplo da cidade de Toritama no qual a totalidade da população trabalha direto ou indiretamente na produção de jeans.

Segundo o LSPA/IBGE (2019), a cidade de Toritama não apresenta nenhum tipo de cultura agrícola, a cidade de Caruaru, apenas milho e feijão, Riacho das Almas cultiva abacaxi, mandioca e feijão, Vertentes, banana, feijão e milho em grão e Surubim, fava, feijão e milho. Todos esses cultivos são realizados de forma muito tímida e familiar. Os municípios têm como principal fonte econômica, a atividade têxtil.

As lavanderias têm uma característica própria, uso de $83 \%$ da força de trabalho masculina tanto na condição de colaborador como na de gestores. Quanto à produção das lavanderias, a quantidade mensal é de 100 mil peças em média. Quanto à quantidade de funcionários, as lavanderias disponibilizam em média 25 funcionários, os quais têm idade média de 32 anos.

Ressalta-se que, a origem dos colaboradores, segundo os dirigentes, $80 \%$ são de pessoas de outras regiões do estado e da Paraíba, com uma maior concentração na mata sul Pernambucana. Esses trabalhadores viviam do cultivo da cana de açúcar e devido ao não funcionamento das usinas saíram para outras cidades em busca de oportunidade.

Outro ponto levantado pelos dirigentes esteve relacionado com a não qualificação dos funcionários no segmento de lavanderias têxteis, $75 \%$ dos colaboradores chegam às lavanderias sem noções básicas da atividade de beneficiamento de jeans atesta a falta de estratégia governamental em valorizar esses APLs. Outra característica apontada pelos respondentes é a forma de contratação dos empregados, uma vez que, $50 \%$ dos contratados são indicados através dos próprios funcionários.

Outra característica do setor, segundo mostram os dados, é que não é exigida escolaridade para o trabalho na atividade, uma vez que, $75 \%$ dos funcionários têm até o ensino fundamental, seja completa ou incompleta e, apenas $23 \%$, têm ensino médio e $2 \%$ nível superior.

Quanto ao grau de escolaridade dos dirigentes, pode-se afirmar que $68 \%$ dos dirigentes têm o ensino médio como nível de escolaridade, seguida de $26 \%$ dos dirigentes com apenas o fundamental e 5\% com nível superior. Quanto à idade, 53\% dos dirigentes têm mais de 40 anos de idade e $47 \%$ têm até 39 anos de idade.

Os gaps existentes entre a produção atual das lavanderias têxteis e o ideal proposto pelos estudos que abordam a $\mathrm{P}+\mathrm{L}$ podem ser visualizados na Tabela 01. Assim, pode-se afirmar que, quanto às dimensões internas as que apresentam menor gap (as que mais se aproximam do modelo $\mathrm{P}+\mathrm{L}$ ) são a saúde do trabalhador contabilizando um gap de 0,77 , conforme Tabela 1 e Figura 1.

Essa dimensão ganha destaque por identificar os cuidados dos dirigentes quanto a disponibilizar equipamentos de proteção individual, do suporte a prática de exercícios físicos e exames laboratoriais, e programa de saúde do trabalhador - ASO (O ASO é o atestado anual exigido por lei que define se o funcionário está apto ou inapto para a realização de suas funções dentro da empresa).

Esse resultado confirma os estudos de Silva, Moraes \& Machado (2015), Quevedo \& Paganini (2017) e Passini, Chagas \& Demarco (2019), os quais concluíram que adotar um programa de $\mathrm{P}+\mathrm{L}$ contribui para a integridade física e mental dos colaboradores, essa se faz através da oferta de equipamentos de proteção individual e programas de saúde do trabalhador integrada às questões econômicas, institucionais e sociais que promovem o equilíbrio socioambiental, além, da contribuição na saúde dos colaboradores.

$\mathrm{O}$ gerenciamento dos resíduos apresentou um gap 1,07 ao identificar que ações como reuso e tratamento de água, monitoramento e classificação dos resíduos, destinação dos resíduos sólidos, lodo das estações de tratamentos de água (ETE), ao aterro sanitário, o uso de filtros para controle de emissão de poluição atmosférica nas caldeiras é realizado por $61 \%$ dos pesquisados, o que tem contribuído para diminuir o gap entre a produção atual e ideal.

Esses resultados corroboram os estudos de Franco \& Arias (2018) e Silva, Fritsch \& Silva (2018), que abordam como fatores importantes para atender ao modelo de $\mathrm{P}+\mathrm{L}$ em uma 
organização a armazenagem e destinação correta dos elementos não reciclados, redução e controle das emissões e resíduos líquidos (efluentes), sólidos e gasosos.

Verifica-se (Tabela 1) que os maiores gaps (próximo de 5,0) são o conhecimento sobre o método de $\mathrm{P}+\mathrm{L}$ (gap 4,23) ao identificar que $80 \%$ dos pesquisados não conhecem nem sabe como aplicar a ferramenta $\mathrm{P}+\mathrm{L}$ na produção, seguido da dimensão colaborador ( $g a p$ de 2,15).

Tabela 1. Os gaps da correlação das dimensões da produção mais limpa atual e ideal. Fonte: Silva, Ribeiro \& Menelau (2019).

\begin{tabular}{lrrlrr}
\hline Dimensões internas & \multicolumn{1}{l}{$\begin{array}{l}\text { Prod. } \\
\text { Atual }\end{array}$} & Gaps & Dimensões externas & $\begin{array}{l}\text { Prod. } \\
\text { Atual }\end{array}$ & Gap \\
\hline Gestão Organizacional & 3,63 & 1,37 & Cliente & 2,64 & 2,36 \\
Colaborador & 2,85 & 2,15 & Fornecedor & 3,07 & 1,93 \\
Conhecimento sobre a P+L & 0,77 & 4,23 & Ambiente Externo & 3,97 & 1,03 \\
Processo produtivo & 3,36 & 1,64 & Matéria-prima & 3,38 & 1,62 \\
Gestão de resíduos & 3,93 & 1,07 & Política e Legislação & 2,77 & 2,23 \\
Saúde do trabalhador & 4,23 & 0,77 & & & \\
\hline
\end{tabular}

Segundo os dados da pesquisa, a conduta da maioria dos profissionais das empresas não está voltada para evitar desperdícios de água, energia e matéria-prima, bem como existe ausência de capacitação, treinamento e programas de educação e conscientização ambiental, além de conhecimentos sobre legislação ambiental.

O gap relacionado com o conhecimento da P+L é considerado pelo CNTL (2003) como o mais gritante, uma vez que se faz necessário conhecer a ferramenta para poder adotá-la de forma eficiente e obter sucesso. O estudo de Costa et al. (2014) corrobora estes achados, ao deixar claro que o conhecimento da ferramenta $\mathrm{P}+\mathrm{L}$ é o ponto chave para a obtenção de ganhos econômicos, sociais e ambientais.
Silva, Moraes \& Machado (2015) afirmam que a $\mathrm{P}+\mathrm{L}$ pode ser uma excelente estratégia para mostrar a credibilidade socioambiental da organização perante a sociedade, além de atender o "novo cliente ecológico".

A Tabela 2 mostra as correlações existentes entre as dimensões organizacionais, processos, fornecedores e ambiente externo com as variáveis: engajamento dos colaboradores para resolver problemas e uso de tecnologia na produção (máquinas de lasers); monitoramento e classificação dos resíduos gerados na produção; preferência dos clientes por produtos biológicos; utilização de meio de mitigação a poluição; parceria com órgãos públicos; quantitativo de funcionários com nível superior e identidade organizacional.

Tabela 2. Correlação entre as dimensões organizacionais, processos, fornecedores e ambiente externo. Fonte: Silva, Ribeiro \& Menelau (2019)

\begin{tabular}{|c|c|c|c|}
\hline Variável & $\begin{array}{l}\text { Coeficiente } \\
\text { Correlação }\end{array}$ & Significância & Dimensões \\
\hline $\begin{array}{l}\text { Engajamento de todos os funcionários para } \\
\text { resolver problemas }\end{array}$ & $\mathrm{R}=0,417$ & $\mathrm{P}=0,009$ & Organizacional \\
\hline $\begin{array}{l}\text { Uso de tecnologia na produção (máquinas de } \\
\text { lasers) }\end{array}$ & $\mathrm{R}=0,428$ & $\mathrm{P}=0,007$ & Processo \\
\hline $\begin{array}{l}\text { Monitoramento e Classificação dos resíduos } \\
\text { gerados na produção }\end{array}$ & $\mathrm{R}=0,438$ & $\mathrm{P}=0,006$ & $\begin{array}{l}\text { Gestão de } \\
\text { resíduos }\end{array}$ \\
\hline $\begin{array}{l}\text { Preferência dos clientes por produtos } \\
\text { biológicos }\end{array}$ & $\mathrm{R}=, 464$ & $\mathrm{P}=0,003$ & Fornecedor \\
\hline Utiliza meio de mitigar a poluição & $\mathrm{R}=0,481$ & $\mathrm{P}=0,002$ & $\begin{array}{l}\text { Ambiente } \\
\text { externo }\end{array}$ \\
\hline Parceria com órgãos públicos & $\mathrm{R}=0,444$ & $\mathrm{P}=0,005$ & Organizacional \\
\hline Funcionário com nível superior & $\mathrm{R}=0,615$ & $\mathrm{P}<0,001$ & Organizacional \\
\hline Identidade organizacional & $\mathrm{R}=0,608$ & $\mathrm{P}<0,001$ & Organizacional \\
\hline
\end{tabular}

Como pode ser observado (Tabela 2), o grau de confiabilidade ficou acima do recomendável $(p<0,010)$ no conjunto de todas as varáveis observáveis para a adoção da $\mathrm{P}+\mathrm{L}$ na organização. 
A análise da Correlação de Spearman identificou correlações entre as variáveis observáveis, podendo ser caracterizado como multicolinearidade.

No entanto, decidiu-se aceitar todas as variáveis com correlação acima de $(\mathrm{R}=0,415)$, pois estas contribuem para discussão teórica referente à adoção da P+L.

A variável funcionários versus nível de superior de escolaridade (graduação) tem correlação $(\mathrm{R}=0,615)$ e grau de significância de $(\mathrm{p}<0,001)$ o que confirmam a relação positiva e moderada entre nível superior e adoção da $\mathrm{P}+\mathrm{L}$ na organização, também indica que o grau de escolaridade exerce uma forte influência sobre a $\mathrm{P}+\mathrm{L}$ evidenciando que quanto maior o nível de escolaridade maior serão as ações de $\mathrm{P}+\mathrm{L}$ e desta forma maior será o sucesso da adoção da $\mathrm{P}+\mathrm{L}$.

O resultado confirma o estudo de Santos (2015) ao diagnosticar que a escolaridade é fator primordial na construção do conhecimento acerca da sustentabilidade bem como no processo de desenvolvimento dessas práticas no ambiente laboral, as correlações das variáveis foram positivas e estatisticamente significativas $(\mathrm{R}=0,97$ e $\mathrm{P}=0,01)$ entre escolaridade e sustentabilidade.

A definição da identidade organizacional pelas organizações também se mostrou com um alto grau de significância $(\mathrm{P}<0,001)$ e correlação $(\mathrm{R}=0,608)$ ao comparar com a adoção da $\mathrm{P}+\mathrm{L}$. Nesse sentido, quanto mais bem definida for a identidade organizacional da empresa, maior será a relação com adoção da $\mathrm{P}+\mathrm{L}$.

As correlações entre o conhecimento da ferramenta $\mathrm{P}+\mathrm{L}$ pelos gestores das empresas têm correlação com o engajamento dos funcionários para resolver problemas, teve correlação de $(\mathrm{R}=0,417)$ e $(\mathrm{P}=0,009)$. Uso de tecnologia na produção (máquinas de lasers) com correlação $(\mathrm{R}=0,428)$ e $(\mathrm{P}=0,007)$. Ou seja, esta correlação é moderada e positiva, assim as duas variáveis crescem na mesma proporção.

O monitoramento e classificação dos resíduos gerados na produção tem correlação de $(\mathrm{R}=0,438)$ e $(\mathrm{P}=0,006)$ ao analisar a correlação entre a preferência dos dirigentes por produtos biológicos e o conhecimento da ferramenta $\mathrm{P}+\mathrm{L}$ ( $\mathrm{R}=0,464)$ e significância de $(\mathrm{P}=0,003)$.

A utilização de meio de mitigar a poluição teve correlação de $(R=0,481)$ e significância de
$(\mathrm{P}=0,002)$ a parceria com órgãos públicos $\mathrm{e}$ conhecer a $\mathrm{P}+\mathrm{L}$ teve correlação $(\mathrm{R}=0,444)$ e significância de $(\mathrm{P}=0,005)$. Essas correlações são classificadas como moderada e positiva, ou seja, são proporcionais, assim quando uma cresce a outra também cresce.

O processo produtivo atual das lavanderias apresentou um gap de 1,64, ou seja, em uma escala de 0 a 5 o atual modelo atende a $\mathrm{P}+\mathrm{L}$ em 3,36 os que levam a identificar que existe uma preocupação por parte dos gestores em reorganizar suas atividades ou modificar a forma de trabalhar para obter melhoras em seus resultados ambientais, adoção de novas soluções para reduzir o custo dos estoques das matérias-primas e pelo entendimento de que, com o atual modelo de produção das empresas, não é possível alcançar equilíbrio entre crescimento econômico e preservação do meio ambiente.

O resultado corrobora o contido no manual de P+L do CNTL (2003) e os estudos de Mohammad et al. (2017), Rahim \& Abdul Raman (2017) e Silva, Fritsch \& Silva (2018), os quais concluíram que o processo voltado para a $\mathrm{P}+\mathrm{L}$ deve contemplar a reciclagem e reuso no ambiente interno, modificações tecnológicas no sistema produtivo, foco na adoção de tecnologias limpas e preventivas.

Os resultados evidenciam que existem correlações moderada e positiva entre a dimensão cliente e condições econômicas das empresas $(\mathrm{R}=0,512) \quad$ e significância de $\quad(\mathrm{P}=0,001), \quad \mathrm{o}$ engajamento dos funcionários com os problemas da empresa teve uma correlação de $(\mathrm{R}=0,482)$ e significância de $(\mathrm{P}=0,002)$, o investimento em capacitação dos colaboradores obteve uma correlação de $(\mathrm{R}=0,513)$ e significância de $(\mathrm{P}=0,001)$, (Tabela 3).

A correlação entre a dimensão cliente e o projeto de educação ambiental foi de $(\mathrm{R}=0,446) \mathrm{e}$ significância $(\mathrm{P}=0,005)$, o conhecimento da lei de Resíduos sólidos apresentou correlação de $(\mathrm{R}=0,429)$ e significância de $(\mathrm{P}=0,007)$. Todas as variáveis analisadas (Tabela 3) apresentam uma correlação positiva e moderada, conforme Pestana \& Gageiro (2003). Um aumento nas dimensões colaboradores, gestão organizacional e legislação leva ao aumento na dimensão cliente e vice-versa.

Tabela 3. Correlação entre as variáveis internas da organização e as dimensões gestão organizacional e legislação. Fonte: Silva, Ribeiro \& Menelau (2019).

\begin{tabular}{|l|l|l|l|}
\hline Variável & $\begin{array}{l}\text { Coeficiente } \\
\text { correlação }\end{array}$ & Significância * & Dimensões \\
\hline Condições econômicas da empresa & $\mathrm{R}=0,512$ & $\mathrm{P}=0,001$ & $\begin{array}{l}\text { Gestão } \\
\text { organizacional }\end{array}$ \\
\hline
\end{tabular}




\begin{tabular}{|c|c|c|c|}
\hline Engajamento de todos para resolver problemas & $\mathrm{R}=0,482$ & $\mathrm{P}=0,002$ & $\begin{array}{l}\text { Gestão } \\
\text { organizacional }\end{array}$ \\
\hline Investimento em capacitação colaborador & $\mathrm{R}=0,513$ & $\mathrm{P}=0,001$ & Colaborador \\
\hline Projeto de educação ambiental & $\mathrm{R}=0,446$ & $\mathrm{P}=0,005$ & $\begin{array}{l}\text { Gestão } \\
\text { organizacional }\end{array}$ \\
\hline Gestão conhecer a lei de Resíduos sólidos & $\mathrm{R}=0,429$ & $P=0,007$ & Legislação \\
\hline $\begin{array}{l}\text { Atender as condições dos padrões de } \\
\text { lançamentos de efluentes no corpo hídrico }\end{array}$ & $R=-0,611$ & $\mathrm{P}<0,001$ & Legislação \\
\hline
\end{tabular}
p valor* $<=1 \%$.

A correlação da dimensão Cliente com o atendimento às condições dos padrões de lançamentos de efluentes no corpo hídrico teve uma correlação forte e negativa de $(\mathrm{R}=-0,611) \mathrm{e}$ significância de $(\mathrm{P}<0,001)$, ou seja, a correlação é moderada e as variáveis analisadas variam em sentido contrário, isto é, as categorias mais elevadas de variável cliente estão associadas a categorias mais baixas da variável atender aos padrões de lançamento de efluentes no corpo hídrico.

$\mathrm{Na}$ análise das dimensões externas, (Figura $1)$, destaca-se como maior gap $(2,36)$ a dimensão "cliente" que segundo os dirigentes, $87 \%$ dos clientes não estão engajados com a sustentabilidade ambiental e nem estão dispostos a pagar por produtos sustentáveis, se eles forem mais caros que os tradicionais. Além de não solicitarem informações sobre os processos produtivos das empresas.

Vale salientar que, os clientes das lavanderias são os confeccionistas de jeans. Para Boons \& Lüdeke-Freund (2013), Mauser et al. (2014) e Passini; Chagas \& Demarco (2019), a participação e a cooperação dos clientes nas mudanças de processos é importante para o equilíbrio socioambiental e saúde pública.

\section{Gaps}

$\longrightarrow$ Modelo de Produção Atual $\longrightarrow$ Modelo de Produção P+L

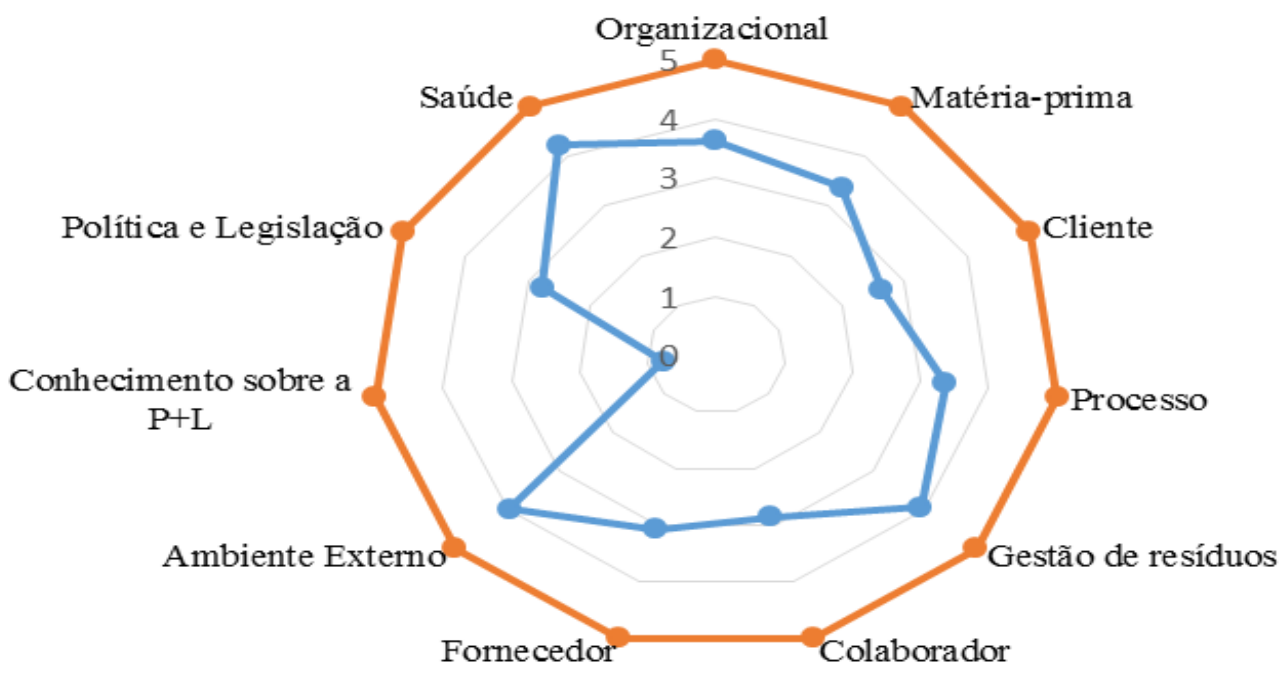

Figura 1. Representação polar dos gaps entre a produção atual e o modelo de Produção Mais Limpa. Fonte: Silva, Ribeiro \& Menelau (2019).

A dimensão política e a legislação (Figura 1) têm um gap de 2,23, o que justifica o fato de $71 \%$ das empresas terem recebido multas ou advertências em 2018.

Esse resultado ressalta a importância da $\mathrm{P}+\mathrm{L}$ abordado no estudo de Quevedo \& Paganini (2017), quando afirma que a ferramenta auxilia no planejamento de políticas públicas promove o incremento na saúde populacional e no meio ambiente de maneira integrada. Silva, Moraes \& Machado (2015) corroboram ao afirmarem que a $\mathrm{P}+\mathrm{L}$ auxilia na redução das despesas com multas e penalidades.

Como pode ser observada (Figura 1), a dimensão ambiente externa apresenta o menor gap 1,03 , entre a produção atual e ideal segundo a $\mathrm{P}+\mathrm{L}$ 
ao identificar que $80 \%$ das empresas pautam suas ações visando à redução dos danos ambientais para a sociedade por meio da busca por soluções para superar problemas ambientais. Além disso, reduzem o monitoramento de água dos rios, poços e cisternas, tal resultado, corrobora o estudo de Silva, Moraes \& Machado (2015), que classifica a $\mathrm{P}+\mathrm{L}$ como uma ferramenta que auxilia na minimização ambiental e criação de uma imagem socioambiental correta.

A matéria-prima também ganha destaque no quesito (segundo menor gap 1,64) de dimensões externas ao identificar que $67 \%$ das empresas utilizam ferramentas para mensurar e controlar do consumo da energia elétrica e consumo de água usadas na produção além de controle químicos de uso restrito (permanganato de sódio).

Passini, Chagas \& Demarco (2019) corroboram ao afirmarem que a $\mathrm{P}+\mathrm{L}$ é um programa que visa a minimização e não geração de resíduos a partir da eliminação dos desperdícios de insumos utilizados nos processos administrativos e produtivos, contribuindo para o gerenciamento da eficiência dos recursos naturais e não naturais.
Regra geral, o gap médio dentre as onze dimensões e a $\mathrm{P}+\mathrm{L}$ foi de 1,54 .

A análise da Correlação de Spearman apresentada na Tabela 4 é de forma moderada e com alto grau de significância. Assim, as condições econômicas e comprometimento dos colaboradores apresentou uma correlação de $\mathrm{R}=0,474$ e significância de $\mathrm{P}=0,003$. A correlação comprometimento dos colaboradores com o investimento em capacitação do colaborador é de $\mathrm{R}=0,632$ e significância de $\mathrm{P}<0,001$.

A parceria com o sistema com instituições de ensino e capacitação e comprometimento dos colaboradores apresentou uma correlação de $(\mathrm{R}=0,459)$ e significância de $(\mathrm{P}=0,004)$, o reuso e tratamento de água apresentou uma correlação de $(\mathrm{R}=0,504)$ e significância de $(\mathrm{P}=0,001)$, ou seja, estas variáveis são correlacionadas de forma positiva e moderada entre si.

Estas variáveis são positivas e quando, as condições econômicas aumentam, as variáveis: colaborador, engajamento dos funcionários e investimento em capacitação e treinamento e reuso de água também aumentam.

Tabela 4. Correlação entre as variáveis comprometimento do colaborador e Produção Mais limpa. Fonte: Silva, Ribeiro \& Menelau (2019).

\begin{tabular}{|l|l|l|l|}
\hline Variável & $\begin{array}{l}\text { Coeficiente } \\
\text { correlação }\end{array}$ & Significância & Dimensões \\
\hline Condições econômicas & 0,474 & $\mathrm{P}=0,003$ & Gestão \\
\hline Investimento em capacitação colaborador & 0,632 & $\mathrm{P}<0,001$ & Colaborador \\
\hline Escolaridade do gestor & $-0,445$ & $\mathrm{P}=0,005$ & Gestão \\
\hline $\begin{array}{l}\text { Parceria com instituições de ensino e } \\
\text { capacitação }\end{array}$ & 0,459 & $\mathrm{P}=0,004$ & Ambiente externo \\
\hline Reuso e tratamento de água & 0,504 & $\mathrm{P}=0,001$ & $\begin{array}{l}\text { Gestão de } \\
\text { resíduos }\end{array}$ \\
\hline
\end{tabular}

p-valor* $<=1 \%$.

Pode-se observar na Tabela 4, que o comprometimento do funcionário com a $\mathrm{P}+\mathrm{L}$ versus nível de escolaridade do gestor $(\mathrm{R}=-0,445)$ estão correlacionados negativamente, ou seja, quanto menor o grau de instrução do gestor, maior o descomprometimento com os princípios da $\mathrm{P}+\mathrm{L}$. o grau de significância $(\mathrm{P}=0,005)$ foi menor de $1 \%$ o que leva a entender que essas duas variáveis se apresentam importante para adoção da $\mathrm{P}+\mathrm{L}$.

A correlação entre comprometimento dos colaboradores e gestor com fundamental apresentase moderada e inversamente proporcional, ou seja, a correlação entre essas duas variáveis é negativa e quando aumenta o comprometimento dos colaboradores diminui a lacuna do nível educacional.

\section{Conclusões}

Os gaps internos mais próximos de zero foram saúde do trabalhador, seguido de gestão de resíduos, que as exigências legais são importantes para o trabalhador, clientes e sociedade.

A variável cliente versus disponibilidade a pagar por um produto sustentável (dimensões externas) apresentou o maior gap (2,36), uma vez que, a maioria dos confeccionistas de jeans não estão dispostos a pagar por um produto mais sustentável, ou seja, o foco do cliente é o preço baixo.

$\mathrm{O}$ atendimento à legislação ambiental também se destaca como o segundo maior gap externo, esse fato fica evidente diante das multas e advertências que as empresas de beneficiamento de jeans sofreram nos últimos anos.

Quanto ao menor gap externo, destaca-se a matéria-prima, uma vez que o setor tem investido no tratamento e reuso da água, além de gerenciar 
os materiais para atender a quantidade de peças de jeans mensais. Vale destacar que essa conformidade não se refere ao uso de produtos biodegradáveis, mas das estratégias de gerenciar a matéria-prima nas organizações.

Como limitação ao estudo, pode-se considerar a falta de interesse da maioria dos proprietários em participarem da pesquisa, seja por falta de conhecimento ou devido ao tempo restrito deles.

Como pesquisa futura recomenda-se analisar as barreiras e fatores de sucesso da $\mathrm{P}+\mathrm{L}$ em todos os elos da cadeia do jeans.

Conclui-se que existem lacunas em todas as dimensões em relação à produção atual das lavanderias têxteis de jeans e a $\mathrm{P}+\mathrm{L}$ abordadas nesse estudo, destacando-se que se faz necessário maior compreensão da ferramenta por parte dos dirigentes e colaboradores, bem como, adoção de políticas educacionais que visem a sustentabilidade para a região em estudo.

\section{Agradecimentos}

À Coordenação de Aperfeiçoamento de Pessoal de Nível Superior (CAPES) pela concessão da bolsa de mestrado que viabilizou a realização deste estudo e ao Programa de Administração e Desenvolvimento Rural - PADR/UFRPE.

\section{Referências}

Amaral, M. C. do; Zonatti, W. F.; Silva, K. L. da; Karam Junior, D.; Amato Neto, J. BaruqueRamos, J. 2018. Reciclagem e reaproveitamento de têxteis industriais no Brasil: estudo de caso e considerações sobre a economia circular. Gestão \& Produção, 25 (3), 431-443.

Araújo W. C. de. 2015. Análise do gerenciamento dos resíduos sólidos gerados pela indústria de confecções do Agreste de Pernambuco. Dissertação. Universidade Federal de Pernambuco. Caruaru, Pernambuco, Brasil. $77 \mathrm{p}$.

Battisti, P. S. S.; Vigorena, D. A. L.; Denuzi, V. S. S.; Knie, D. C. 2015. O uso da tecnologia no tratamento estatístico em pesquisas na área de secretariado executivo. Revista Capital Científico Eletrônica, 13, (1), 77-94. doi: 10.5935/2177-4153.20150005.

Bezerra, A. S.; Freitas, L. S. 2013. Avaliação das atividades de uma lavanderia industrial à luz da P+L no polo de confecções de Santa Cruz do Capibaribe - PE. Anais XVI SEMEAD Seminários em Administração. Universidade de São Paulo, 2013, São Paulo, Brasil, FEAUSP. 16, (1), 16-31.
Bezerra, F. F. N.; Monteiro, M. S. L. 2009. Sistema de gestão ambiental ou P+L? Um estudo de caso nas empresas de confecção com lavanderia, Teresina, Piaui. REDE-Revista Eletrônica do Prodema, 3, (1), 42-61.

Buss, M. V.; Ribeiro, E. F.; Schneider, I. A. H.; Menezes, J. C. S. dos S. 2015. Tratamento dos efluentes de uma lavanderia industrial: avaliação da capacidade de diferentes processos de tratamento. Revista de Engenharia Civil IMED, 2, (1), 2-10.

CNTL - Centro Nacional de Tecnologias Limpas. $\mathrm{P}+\mathrm{L}$ (2003). Disponível em: http://www.senairs.org.br/cntl/.

Costa, E. M.; Oliveira Neto, G. C. de; Lucato, W. C. 2014. Avaliação da Eco eficiência da Implantação da $\mathrm{P}+\mathrm{L}$ em uma Indústria Têxtil. Evento do XI simpósio de excelência gestão e tecnologia - SEGeT, Rio de janeiro.

Fonseca, J. J. S. 2002. Metodologia da pesquisa científica. Fortaleza, UECE, Ceará. 120p.

Fonseca, R. A.; Lima, A. B.; Rezende, J. L. P. de; Nazareth, L. G. C.; Santiago, T. M. O. 2013. P+L: uma nova estratégia de produção. Evento X SEGET- Gestão e Tecnologia para a Competitividade, Associação Educacional Dom Bosco-AEDB, Resende, Rio de Janeiro, 10, (1), 1-11.

Franco, C.; Arias, J. L. 2018. Sistemas de gestión ambiental y procesos de producción más limpia en empresas del sector productivo de Pereira y Dosquebradas. Entre Ciencia e Ingeniería, 12, (23), 140 - 146.

Gil, A. 2007. Como elaborar projetos de pesquisa. 4. ed. São Paulo: Atlas. 176p.

Hoof, B. V. 2014. Organizational learning in cleaner production among Mexican supply networks. Journal of Cleaner Production, 64, (1), 115-124.

Lopes, C. S. D. 2011. Análise ambiental da fase de acabamento do jeans. Revista de Saúde, Meio Ambiente e Sustentabilidade, 6, (3), 87-102.

Malhotra, N. K. 2012. Pesquisa de Marketing: uma orientação aplicada. 6. ed. Porto Alegre: Bookman. 720p.

Nascimento, S. 2021. Design de Moda para Sustentabilidade: uma proposta de aproveitamento de retalhos da indústria têxtil de Uberlândia - MG. 2021. Universidade Federal de Uberlândia, Uberlândia, Brasil, $52 \mathrm{p}$.

Ortolano, L.; Trianab, E.S.; Afzalc, J.; Alid, C.L.; Rebellónb, S. A. 2014. Cleaner production in Pakistan's leather and textile sectors. Journal of Cleaner Production, 68, (1), 121-129.

Passini, A. F. C.; Chagas, P.; Demarco, J. de O. 2019. Opções de $\mathrm{P}+\mathrm{L}$ para uma padaria de 
pequeno porte. Revista Gestão e Produção, 26, (3), 1-11. Doi: 10.1590/0104-530x361319.

Pimenta, H. C. D.; Gouvinhas, R. P. 2012. Cleaner production as corporate sustainability tool: a study within companies from Rio Grande do Norte State. Revista Científica Eletrônica de Engenharia da Produção, 22, (3), 462-476. Doi: 10.1590/S0103- 65132012005000043

Quevedo, C. M. G. de; Paganini, W. da S. A. 2017. Gestão do fósforo nas águas: estudo de caso do Rio Tietê, Brasil. Revista Ambiente \& Água, 12, (6), 902-917. Doi: 10.4136/ambiagua.1977.

Rahman, N. A. A.; Mohamad, M. F.; Rahim, S. A.; Hassan, R.; Ahmad, M. D.; Kadir, S. A. 2017. Shippers perceptions of aviation logistics service quality (LSQ) of air freight provider. Journal of Engineering and Applied Sciences, 12, (3), 699-704.

Santos, M. F.; Santos, R. S.; Beretta, M. 2010. Reuso de Efluentes em Atividades Industriais, Escola Politécnica-UFBA, Relatório técnico, Bahia, Brasil.

Santos, J. G.; Cutolo, S. A.; Piveli, R. P.; Campos, F.; Sundefeld, G.; Sousa, T. S. 2012. Análise parasitológica em efluentes de estações de tratamento de águas residuárias. Revista de Patologia Tropical, 41, (3), 319-336.

Santos, B. M.; Godoy, L. P.; Guillet, V. M.; Prato, C. R.; Sagrrillo, M. 2018. Diagnóstico e reflexão sobre os processos de $\mathrm{P}+\mathrm{L}$ numa empresa do ramo metal- mecânico. Revista Metropolitana de Sustentabilidade, 8, (2), 3348.

Silva Filho, J. C. G. 2003. O uso da P+L como ferramenta de gestão ambiental de melhoria contínua: estudo de caso em uma empresa fabricante de embalagem de papel no estado de Pernambuco. Dissertação. Universidade Federal de Pernambuco, Recife, Brasil. 167p.

Silva, A. L. E.; Moraes, J. A. R.; Machado E. L. 2015. Proposta de $\mathrm{P}+\mathrm{L}$ voltada às práticas de ecodesign e logística reversa. Revista Engenharia Sanitária Ambiental, 20, (1), 2937.

Silva, L.; Fritsch, R. L. C.; Silva, V. M. 2018. Metodologia da Produção Mais Limpa $(\mathrm{P}+\mathrm{L})$ : abordagem conceitual e casos. Ciência e Tecnologia, 2, (2), 49-56.

Silva, M. D. F.; Menelau, A. S. 2021. Cultura organizacional como fator determinante para a adoção da Produção Mais Limpa. Journal of Environmental Analysis and Progress, 6, (1), 001-011.

Silva, M. F.; Oliveira, C. N. O.; Ribeiro, A. R. B.; Correia, R. C.; Araújo, J. L. P. 2018. P+L na cultura do melão em Petrolina - PE: uma parceria entre a EMBRAPA semiárido e os produtores locais. Ciência Agrícola, 16, (1), $37-41$.

Vergara, S. C. 2014. Projetos e relatórios de pesquisa em administração. Editora Atlas, São Paulo, 145p. 